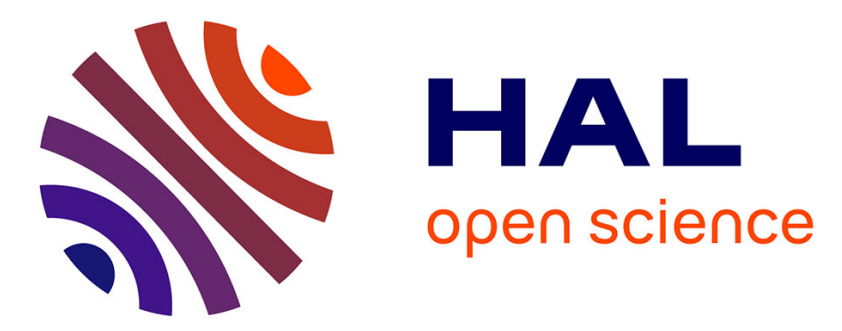

\title{
P2 hierarchical decomposition procedure: application to irrigation strategies design
}

Olivier Crespo, Jacques-Eric Bergez, Frederick Garcia

\section{To cite this version:}

Olivier Crespo, Jacques-Eric Bergez, Frederick Garcia. P2 hierarchical decomposition procedure: application to irrigation strategies design. Operational Research, 2011, 11 (1), pp.19-39. 10.1007/s12351-009-0040-z . hal-00745693

\section{HAL Id: hal-00745693 \\ https://hal.science/hal-00745693}

Submitted on 26 Oct 2012

HAL is a multi-disciplinary open access archive for the deposit and dissemination of scientific research documents, whether they are published or not. The documents may come from teaching and research institutions in France or abroad, or from public or private research centers.
L'archive ouverte pluridisciplinaire HAL, est destinée au dépôt et à la diffusion de documents scientifiques de niveau recherche, publiés ou non, émanant des établissements d'enseignement et de recherche français ou étrangers, des laboratoires publics ou privés. 


\title{
$P 2$ hierarchical decomposition procedure: application to irrigation strategies design
}

\author{
O. Crespo · J.E. Bergez · F. Garcia
}

Received: date / Accepted: date

\begin{abstract}
Optimization by simulation of agricultural practices can help to improve irrigation water use efficiency. This work introduces an efficient hierarchical decomposition method to design irrigation management strategies that is modelled as a continuous stochastic problem. Various combinations of selection (greedy, Pareto-based), division (middle, pivot, maximization) and evaluation techniques (global, standard deviation) were tested. We present results of an 8-continuous-parameter irrigation strategies design. Two criteria were chosen to evaluate the different combinations: the achieved direct margin, and the number of simulation runs that were needed to reach it. Selection techniques impacted the resolution time, while the evaluation techniques impacted the direct margin efficiency. Based on the two former criteria, the trade-off combination of greedy selection, pivot partition and average value evaluation appeared to be the most efficient to design irrigation strategies.
\end{abstract}

Keywords Simulation optimization · crop model · irrigation management

\section{Introduction}

According to the FAO, one of the most important challenges for the agriculture in the decades to come is to increase agricultural production to feed the increasing world population. Much of this increase has to come from an intensive and scientifically-based agriculture, supported by irrigation (FAO (2002)). However, in many countries, water resources

O. Crespo

CSAG, EnGEo Department, University of Cape Town,

Private Bag X3, Rondebosch 7701, Cape Town, South Africa

Tel.: +27 216503164 , Fax: +27 216505773

E-mail: olivier.crespo@csag.uct.ac.za

J.E. Bergez

INRA, UMR 1248 AGIR, BP 52627, 31320 Castanet Tolosan, France

Tel.: +33 561285 037, Fax: +33 561735537

E-mail: jbergez@toulouse.inra.fr

F. Garcia

INRA, Unité Biométrie et Intelligence Artificielle, BP 52627, 31320 Castanet Tolosan, France

Tel.: +33 561285 283, Fax: +33 561285335

E-mail: fgarcia@toulouse.inra.fr 
are already very heavily exploited (Smith (2000)). Where irrigated agriculture is developed, water used for irrigation can represent more than $90 \%$ of the water consumption. Thus we investigate the possibility of increasing the agricultural production by increasing the efficiency of irrigation water use without increasing the quantity of water used.

Agricultural practices have been modelled for some time using decision rules (Papy (2000), Aubry et al (1998), Shaffer and Brodahl (1998)). Such modeling allows uncertain events such as weather to modify planned technical action on the biophysical system in an adaptive way. For example, the weather influences the sowing date. This type of modeling aims at representing the farmer's behavior: actions are decided with regard to both constraints and goals, and are in addition modified depending on context and local conditions. MODERATO (Bergez et al (2001a)) has been developed using such a modeling framework. The cultural operations are decided with elementary conditional rules. These rules involve condition parameters such as cumulative thermal units, water deficit or irrigation amounts. Its purpose is to evaluate current irrigation strategies for corn and to propose improved strategies.

To optimize management strategies, two main approaches can be applied: either controlbased optimization or simulation-based optimization (Bergez et al (2006)). The former approach was used with MODERATO, by testing stochastic dynamic programming and reinforcement learning methods for identifying optimal decision rules (Bergez et al (2001b)). Input variables to the optimization problem were the condition parameter of the decision rules. It gave poor results due to the complexity of the problem and the large variable space to optimize. The simulation-based optimization approach deals with large continuous parameters problems and involves explicit techniques to handle with uncertainty. Various methods have been introduced and developed in this field. Reviews from Andradóttir (1998), Ólafsson and Kim (2002) or Fu et al (2005) provide a clear insight of this diversity: gradient-based procedures, stochastic approximation, sample path optimization, response surface methodology, ranking \& selection, and a few branching approaches dealing with continuous or discrete input variable problems. These methods include stochastic search techniques moving from a current best solution to the next, or deterministic search techniques relying on an approximation of either the response or the objective function.

Among them, a few branching procedures have been introduced in which the feasible region is iteratively broken down before separating promising subsets from unpromising ones. For example, Norkin et al (1994) proposed a version of the branch-and-bound method for discrete parameter optimization where promising and unpromising regions are selected, based on objective function value bounds. This method has recently been introduced for continuous parameter optimization. As another example, $P B n B$ from Prasetio et al (2004) seeks to identify the most promising sub-region containing a desired solution by an iterative pruning-or-branching procedure. Branching action is performed according to the probability of reaching a targeted function measurement, while pruning is performed according to the significant difference of statistical tests between the most- and least-promising regions.

The aim of this paper is to present the $P 2$ algorithm, a development of $P 2 p$ introduced in Bergez et al (2004). $P 2$ is a hierarchical decomposition procedure dedicated to continuous parameter optimization, and is one of the simulation-based optimization methods. Promising regions are selected by a heuristic, relying both on the average value and on the standard deviation of simulation responses. The promising region is then divided into two parts, and each of them is evaluated by simulation. Different options for the three steps of selection, division and evaluation were tested on a corn crop irrigation management problem in order to identify the combination of techniques that lead to the most efficient algorithm. 
We first describe the principle of the $P 2$ algorithm and the various techniques that are tested for selection, division and evaluation. In section 3, we give some information on the MODERATO bio-decisional simulation model used to simulate the irrigation strategies and we set up the simulation experiments. We present the results of the combinations of techniques and we eventually discuss the best $P 2$ algorithm options and the optimized irrigation strategies.

\section{From $P 2 p$ to $P 2$ algorithm}

The $P 2 p$ algorithm is based on the DIRECT (Jones et al (1993)) and the MCS (Huyer and Neumaier (1999)) algorithms which have been developed for deterministic optimization. The $P 2 p$ algorithm is dedicated to large continuous input variable problems. It includes a single objective function, which can be the combination of weighted multiple objectives. $P 2 p$ belongs to the family of stochastic branching methods, like stochastic branch-andbound or nested partitions methods. It is based on a hierarchical decomposition of the decision space into a binary tree.

This algorithm is dedicated to the optimization problem

$$
\max _{\theta \in \Theta} J(\theta),
$$

where $\Theta$ is the set of possible decisions, and $J(\theta)$ is a performance function that can not be computed analytically. The evaluation of $J(\theta)$ thus relies only on the responses of stochastic simulation runs. The formulation of this kind of optimization involves input continuous $D$-dimensional variables $\theta$ constrained to be contained within a feasible region $\Theta \subset \mathbb{R}^{D}$. An objective function is defined on these variables, such that $J: \theta \rightarrow \mathbb{R}$, where $J(\theta)=$ $E[L(\theta, \omega)]$ and $\omega$ is the uncontrollable input variables vector of the stochastic system. The general formulation of simulation-based optimization is:

$$
\max _{\theta \in \Theta} J(\theta)=E[L(\theta, \omega)]
$$

The decision space $\Theta$ is a hyper-rectangle, or region, of $\mathbb{R}^{D}$. The $P 2$ optimization aims at finding small hyper-rectangles included in $\Theta$ which contain the decision vector that maximizes the expected value of $L(\theta, \omega)$. We assume a minimal size of these small regions, defined by the user for every dimension as the width $p_{d}^{\text {step }}$ of the dimension $d \in D$.

Let us call pending regions the regions that are still divisible (or breakable). The principle of the $P 2$ algorithm is described in the Algorithm 1. Initialization allocates the initial decision space as the single pending region of the list pending $R L$ of all the pending regions. The first step consists of SELECTING out from the pending regions list the region which is potentially optimal: we call it the promising region. The second step DIVIDES this promising region into two parts. These two parts are offspring regions and are collected in the offspringRL list. During the third step, each of these regions is sampled, simulated and indices are EVALUATED. Eventually, the pending regions list is updated, and the three previous steps are repeated until stopping criteria are reached or the pending regions list is empty. The $P 2$ algorithm main stopping criterion is achieved when there is no more pending region, i.e. there is no more potentially optimal region which could still be divided for further exploration. This division limit is explain in the next section 2.2. For practical reasons, time limits and/or simulation number limits are usually added to the previous stopping criterion. 


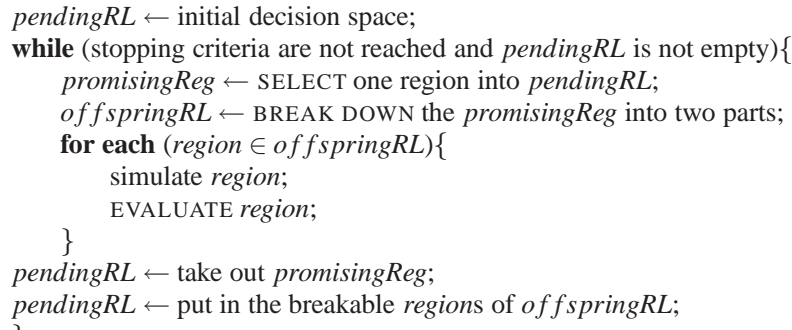

Algorithm 1 The P2 algorithm and its three main steps.

tree search
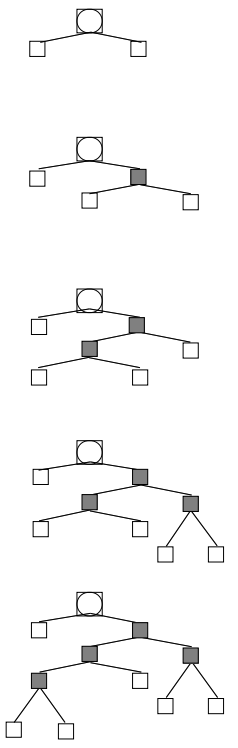

iteration 1

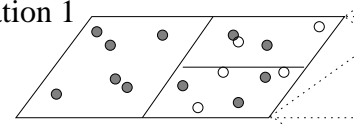

iteration 2

decision space

iteration 0
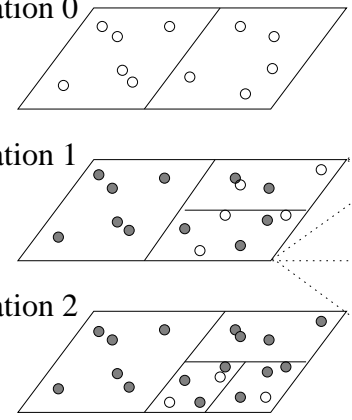

iteration 3

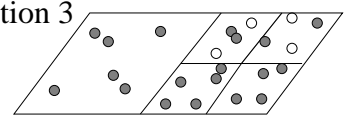

iteration 4

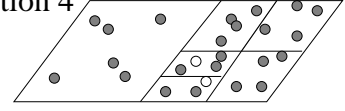

main loop process
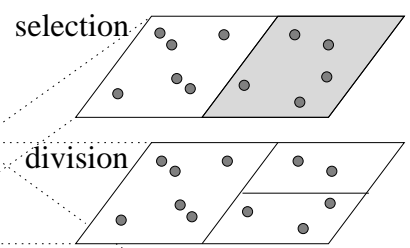

evaluation $\% \circ$

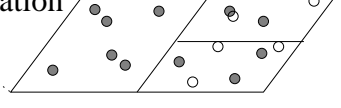

Fig. 1 P2 process illustration: left hand side displays the tree construction related to the decision space exploration, and the right hand side shows iteration 1.

Figure 1 illustrates the three main steps considering a 2-dimensional decision space related to the decision tree produced. The $P 2 p$ algorithm included the $\beta$-selection, middle partition, average value and standard deviation evaluations. The major improvements leading from $P 2 p$ to $P 2$ consisted in proposing, testing and selecting combinations of new techniques.

\subsection{Selecting the promising region}

The selection step is involved in the process efficiency according to its ability to lead straight to the optimal region. At each iteration, the promising region to be selected is one of the pending regions. Each pending region is sampled, simulated and indices are computed dur- 
ing the evaluation step. The focus here is to choose one promising region $r_{*}$ within the pending regions set, based on these indices. Three selection techniques have been tested, based on two indices: the average value and the standard deviation of the simulated responses. We extended the consideration of these two indices to score $_{1}$ and score $_{2}$ which could be either the average value, the standard deviation or the maximum, the minimum or the median value for example.

a. The greedy selection consists in choosing the promising region considering only the expected value criterion to be optimized. Considering score $_{1}$ as a generalization of the criterion to be optimized and maximized, the promising region $r_{*}$ will be chosen such that $r_{*}=\arg \max _{r}\left[\right.$ score $\left._{1}(r)\right]$.

b. We propose a trade-off between greedy and systematic search. The $\beta$-selection takes into account both the average value of the region and the standard deviation of the sampled decision vectors. Let us define score $_{1}$ as the average value and score $_{2}$ as the standard deviation. The figure 2 represents the set of the convex non dominated ${ }^{1}$ regions, in which we select the promising one. We use an approach based on multiobjective optimization, which looks for regions with a large standard deviation when regions are large, and for regions with a large average value when regions are small (Bergez et al (2004)). Considering score $_{1}$ and score $_{2}$ as a generalization of the criteria to be optimized and maximized, let us define $\beta$ as following.

$$
\beta(r)=\text { score }_{2}(r)+\text { score }_{1}(r) \cdot \tan (\gamma) \text { with } \gamma=\frac{\text { current depth }}{\text { maximum depth }+\varepsilon} \cdot \frac{\pi}{2}
$$

current depth is the tree depth of the last region of interest, and maximum depth is the depth of an unbreakable region assuming middle partitioning. $\varepsilon$ is an infinite small value excluding $\pi / 2$ from reachable values. As $\gamma \in[0 . . \pi / 2[$ is tree depth dependant, maximizing $\beta$ is selecting one region among all non dominated ones as shown on figure 2. Then the promising region $r_{*}$ will be chosen among all the pending regions $r$ such that $r_{*}=\arg \max _{r} \beta(r)$.

c. The previous approach can only reach the convex non dominated front. In order to generalize the method to non convex front, the $\operatorname{Conc} \beta$-selection combines the previous method with non convex dominance ${ }^{1}$. The selection technique still gives priority to the standard deviation when regions are large, and to average value when regions are small. In that case only the generalized criteria score $_{1}$ and score $_{2}$ have to be minimized. $\operatorname{proj}_{1}$ and $\operatorname{proj}_{2}$ are the projections of respectively score $_{1}$ and score $_{2}$ onto the line angled $(\pi / 2)-\gamma$ such that $\operatorname{proj}_{1}(r)=\operatorname{score}_{1}(r) * \cos \left(\frac{\pi}{2}-\gamma\right)$ and $\operatorname{proj}_{2}(r)=$ score $_{2}(r) * \sin \left(\frac{\pi}{2}-\gamma\right)$. The promising region $r_{*}$ is still part of the Pareto's set and will be chosen such that

$$
r_{*}=\underset{r}{\arg \max }\left(\min \left[\operatorname{proj}_{1}(r), \operatorname{proj}_{2}(r)\right]\right) .
$$

The figure 3 describe the conc $\beta$-selection technique. Depending on the current depth it allows to select pending regions from the non-convex Pareto front ${ }^{1}$.

\footnotetext{
${ }^{1}$ See for example Ehrgott (2005) for dominance definitions.
} 


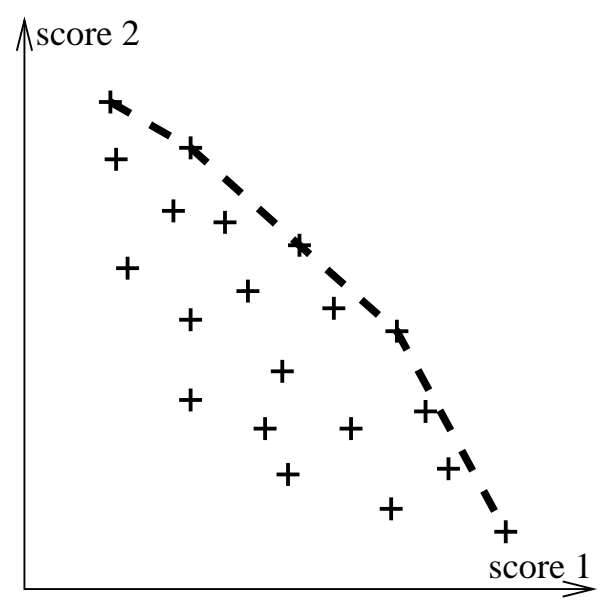

Fig. 2 Some of the pending regions are incorporated in the convex non dominated front. The promising region is one of these. N.B. score ${ }_{1}$ and score 2 have to be maximized.

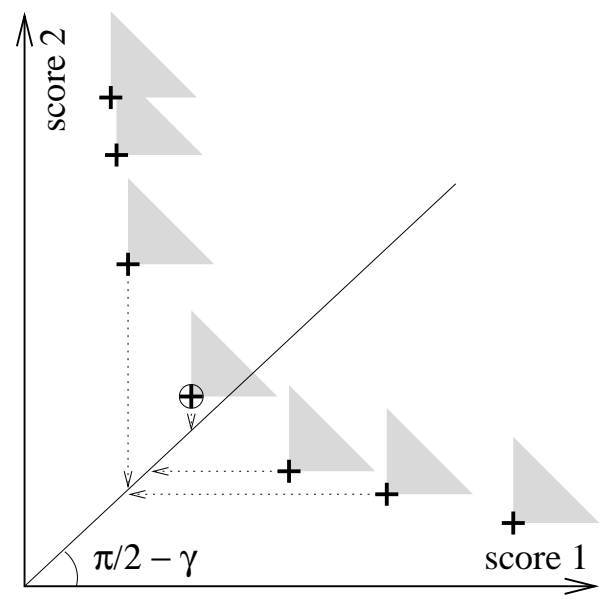

Fig. 3 Conc $\beta$-selection visual description. N.B. In that case only, score ${ }_{1}$ and score $_{2}$ have to be minimized.

\subsection{Breaking down the promising region}

The division step is involved in the resolution time that is needed and in the global optimization efficiency: producing small regions could trap the process in local optima, and producing large regions could be time-consuming.

We proposed in $P 2 p$ to break the $D$-dimensional promising region into $2^{D}$ sub-regions. The depth of the decision tree to reach an unbreakable region is small, but at every nodes one region is taken out from the pending regions list while potentially $2^{D}$ are put in. The number of simulations needed to evaluate every new pending regions is then enormously expended at every division. We chose in the $P 2$ method to cut down the $D$-dimensional promising region into only two parts to save simulations. Hence we first need to choose the parameter 
$p_{*}$ to be cut. We proposed to choose this parameter with the largest relative range such that

$$
p_{*}=\underset{d=1}{\operatorname{Drg} \min }\left(\left(p_{d}^{\oplus}-p_{d}^{\ominus}\right) / p_{d}^{\text {step }}\right)
$$

where $p_{d}^{\oplus}\left(p_{d}^{\ominus}\right)$ is the upper (lower) bound of dimension $d$ and $p_{d}^{\text {step }}$ is its smallest feasible range. Thus three division techniques have been tested.

a. The equal partitioning is the easiest way to proceed. Without taking into account the results from samples, the parameter $p_{*}$ is simply cut down right in the middle, producing two equal sub-regions.

Instead of blindly producing two sub-regions, two other techniques aim at producing two regions as different as possible.

b. The pivot partition chooses the decision vector which divides weak from better responses. The selection of the pivot vector is based on vector indices related to the probability of being the best representation of the threshold dividing the $\gamma \%$ of the 'best' responses from the $1-\gamma \%$ of the 'worst' responses. Two user defined indices $\gamma r$ and $\gamma_{s}$ are used.

c. The maximization of the difference technique is based on a binary classification and regression tree field (Breiman (1996)). The best split is taken as the maximizer of a "goodness-of-split" function and is chosen as the one maximizing the difference between potential sub-region indices $\gamma r$.

\subsection{Evaluating eligible regions}

The system performance is not directly available and we therefore rely on sampled points $L(\theta, \omega)$ of the region of interest. $N$ sampled point vectors $\theta_{i}(i \in[1 . . N])$ are simulated $M$ times $(j \in[1 . . M])$ in each new pending region. $N$ is linearly related to the region width and $M$ is a parameter to be chosen. The aim is to compute, based on $N \times M$ sampled performance measures, the indices of the pending regions that are used in the next iteration to select the promising region.

a. As the optimization definition 2 involves the expected value of the sampled performance measures, we first compute the average value $\hat{J}(\theta)$ as an approximation of $J(\theta)=$ $E[L(\theta, \omega)]$.

$$
J(\theta) \approx \hat{J}(\theta)=\frac{1}{N} \frac{1}{M} \sum_{i=1}^{N} \sum_{j=1}^{M} L\left(\theta_{i}, \omega_{j}\right) .
$$

b. The global standard deviation $V_{g}$ can be used in the selection techniques involving two indices.

$$
V_{g}=\frac{1}{N} \frac{1}{M} \sum_{i=1}^{N} \sum_{j=1}^{M}\left[\hat{J}(\theta)-L\left(\theta_{i}, \omega_{j}\right)\right]^{2} .
$$

c. If we consider the sampled performance measures as disturbed responses of the objective function $J$, then the idea of the third proposition is to use the standard deviation of the objective function without considering the standard deviation of the disturbance. We tested two indices $V_{p 1}$ and $V_{p 2}$. They are computed to focus on the standard deviation due to the controllable input parameters $(\theta)$, excluding the standard deviation due to the uncontrollable input parameters $(\omega)$. The aim of this paper is not to establish the formulation of these standard deviations. Nonetheless, $V_{p 1}$ is based on the hypothesis 
that the response $L\left(\theta_{i}, \omega_{j}\right)$ depends only on the $\theta_{i}$ and $\omega_{j}$ effects, although $V_{p 2}$ is based on the hypothesis that the response $L\left(\theta_{i}, \omega_{j}\right)$ depends on $\theta_{i}, \omega_{j}$ and the residue due to the combination of $\theta_{i}$ with $\omega_{j}$.

\section{Choosing algorithm options by application to irrigation management}

We have presented various techniques of selection, division and evaluation of decision hyper-rectangles. The aim of this section is to present the experimental plan which guided our choice of the best combination of technique. First we introduce the irrigation strategy simulator and the irrigation strategies design application. Then, we define the system to be optimized and the experiments we ran.

\subsection{MODERATO Simulator}

MODERATO (Bergez et al (2001a)) is a model aimed at evaluating current irrigation strategies for corn and at proposing improved strategies. It combines a dynamic and biophysical corn crop model with a dynamic decision model. The crop model is described in Wallach et al (2001). The decision model consists of a set of decision rules for different management decisions, and especially for irrigation management decisions. The crop model and the decision model interact every day. The crop model updates the state variables each day and passes their values to the decision model together with the explanatory variables of that day. Within that collection of variables are the indicators of the decision rules. Then, the decision model evaluates the decision rules to decide if a management action is to be taken. If so, this information is passed back to the crop model (for example, amount of water or sowing density).

The timing of irrigation includes for example these rules:

Starting irrigation This rule determines the starting day to begin irrigation during the growing season and the water amount for the first irrigation round.

Next irrigation round This rule is invoked after a round of irrigation has been terminated. It determines when to start the next round and the irrigation amount for rounds after the first.

Stopping irrigation This rule is invoked at the end of an irrigation round. It has one of these three conclusions: either (1) the previous round of irrigation was the last, or (2) another round of irrigation is to be performed and will be the last, or (3) we will re-invoke this rule after another round of irrigation. Granting that the next round is the last, the amount of irrigation is given.

Many of the rules in MODERATO are based on the general form: - if (cond_1 $a \mathrm{~V}$ cond_1 $1 b) \wedge($ cond_2 $2 a \vee$ cond_2 $2 b)$ then decision; define amount; - where cond_1 $a$ and $1 b$ concern crop development while cond $\_2 a$ and $2 b$ refer to water status in the soil. The first condition in each pair (cond_ $1 a$ and $2 a$ ) uses meteorological variables as indicator variables while the second condition in each pair is based on state variables. The user can choose to ignore one of the two conditions in each part of the premise.

\subsection{Case study}

The comparison between the developed options was performed on an eight-parameter strategy as follows. 
The main irrigation period starts from $\mathbf{T 1}$ as soon as the soil water deficit reaches D1. An amount $\mathbf{I} 1$ is applied. Once an irrigation cycle ends, a new cycle starts when the soil water deficit reaches D2. An amount $\mathbf{I} 2$ is applied. For the irrigation cycle following T3, if the soil water deficit is greater than $\mathbf{D 3}$ before this irrigation cycle starts, a last irrigation cycle is performed; otherwise the irrigation program ends. An amount $\mathbf{I} \mathbf{3}$ is applied.

\begin{tabular}{|c|c|}
\hline Operation & Rules \\
\hline Sowing & $\begin{array}{l}\text { Sowing is between } 20 \text { April and } 30 \text { May as soon as the cumulative rainfall during the } \\
\text { previous } 3 \text { days is less than } 15 \mathrm{~mm} \text {. Variety Cécilia is sown at } 80000 \text { plants/ha. Cécilia } \\
\text { is a late growing variety requiring } 1045 \text { accumulated thermal units (ATU) from sowing to } \\
\text { flowering and } 1990 \text { ATU from sowing to maturity ( } 35 \% \text { grain humidity). }\end{array}$ \\
\hline Fertilization & A single application of $200 \mathrm{~kg} / \mathrm{ha}$ of nitrogen is made at sowing. \\
\hline Harvest & $\begin{array}{l}\text { The crop is harvested when grain moisture content reaches } 20 \% \text { or accumulated thermal } \\
\text { units since sowing reach } 2100 \text { ATU and if the cumulative rainfall during the previous } 3 \\
\text { days is less than } 15 \mathrm{~mm} \text {. In any case, the crop must be harvested before } 15 \text { October. }\end{array}$ \\
\hline \multicolumn{2}{|r|}{ ( } \\
\hline & $\begin{array}{l}\text { Sowing Irrigation to facilitate plant emergence (caused either by dryness or crust created } \\
\text { by heavy rainfall on silty soil) is not taken into account, nor irrigation to dissolve } \\
\text { fertilizer. }\end{array}$ \\
\hline & Starting irrigation Part of the optimization process. \\
\hline & Next irrigation round Part of the optimization process \\
\hline & $\begin{array}{l}\text { Delay irrigation Precipitation delays irrigation. When the cumulative rainfall over the } 5 \\
\text { previous days is more than } 10 \mathrm{~mm} \text {, one day delay is applied for every } 4 \mathrm{~mm} \text {. The } \\
\text { delay cannot exceed } 7 \text { consecutive days. }\end{array}$ \\
\hline & Stopping irrigation Part of the optimization process \\
\hline
\end{tabular}

Table 2 General description of the strategy simulated.

The other cultural operations are given in table 2. The irrigation equipment used for the study allows a $3.5 \mathrm{~mm} /$ day flow rate. A $180 \mathrm{~mm}$ limitation of available water is applied. No flow rate restrictions are imposed during summer except those due to the equipment.

All simulations were performed using a medium clay-silt soil : $0.8 \mathrm{~m}$ deep, with clay accumulation at depth, locally called "Boulbènes moyennes" (fluvisol). This type of soil is representative of a large area of the Midi-Pyrénées and has a $150 \mathrm{~mm}$ cumulative available water capacity. The soil was assumed to be at field capacity at the beginning of the simulation, namely the $1^{\text {st }}$ of January. Climates used are part of the observed weathers recorded at the Toulouse-Blagnac meteorologic station within 1949 and 1997. On average, July and August receive a total of $92 \mathrm{~mm}$ rainfall and the cumulative potential evapotranspiration $\left(\mathrm{ET}_{0}\right)$ is $290 \mathrm{~mm}$. The average evaporative moisture deficit (ET $\mathrm{E}_{0}$ minus rainfall) for this two-month period is around $200 \mathrm{~mm}$. However, there is a large variation in rainfall during the two summer months as it ranges from 30 to $240 \mathrm{~mm}$, underlining the unpredictable nature of rainfall in the area. Cumulative $\mathrm{ET}_{0}$ is less variable, ranging from 235 to $372 \mathrm{~mm}$. The objective function to be maximized is the expectation of the direct margin (i.e. the gross margin minus specific costs for a given activity, here irrigation). The direct margin for irrigation can be written as a weighted sum of multiple criteria:

$$
L\left(\theta_{i}, \omega_{j}\right)=a\left(\theta_{i}, \omega_{j}\right) \cdot B-\left[C+d\left(\theta_{i}, \omega_{j}\right) \cdot E+f\left(\theta_{i}, \omega_{j}\right) \cdot G\right]
$$

where $L\left(\theta_{i}, \omega_{j}\right)$ is the direct margin for climate $\omega_{j}$ and the strategy $\theta_{i}, a\left(\theta_{i}, \omega_{j}\right)$ is the grain yield obtained under climate $\omega_{j}$ and using the strategy $\theta_{i}, B$ is the selling price for corn, 
$C$ is the operational costs for corn production, $d\left(\theta_{i}, \omega_{j}\right)$ is the amount of water used under climate $\omega_{j}$ and using the strategy $\theta_{i}, E$ is the cost of irrigation water, $f\left(\theta_{i}, \omega_{j}\right)$ is the number of irrigation cycles performed under climate $\omega_{j}$ and using the strategy $\theta_{i}$ and $G$ is the cost of carrying out a new irrigation cycle. The average selling price for maize (grain) is assumed to be $106.71 € / \mathrm{Mg}$ in the Toulouse area. Operational costs (seed, weeding, fertilizer, insurance) are assumed to total $327.77 € /$ ha. The cost of irrigation water is assumed to be $0.76 € / \mathrm{mm}$ and the setting up of a new irrigation cycle is assumed to be $7.62 €$.

\subsection{Experiments}

Simulations were run with a Bi-processor of $3 \mathrm{GHz}$ each, and 2 Go of RAM with Windows XP operating system. Optimization took about 3 hours and 40 minutes for 2 million of simulation runs including less than 9 seconds for the $P 2$ procedure.

We took into account 10 replications for each alternative. We particularly focused on robustness of an alternative through the minimum, maximum and average direct margin values of these 10 replications. The initial feasible region is defined in table 3 as the ranges of the different parameters from the strategy described in section 3.2.

\begin{tabular}{rlcccc}
\hline Names & Meaning & unit & min & max & step \\
\hline T1 & Accumulated thermal unit to start the irrigation program & ${ }^{\circ}$ C.day & 200 & 1250 & 5 \\
D1 & Soil water deficit to start the irrigation & $\mathrm{mm}$ & 20 & 150 & 3 \\
I1 & Irrigation applied at the first irrigation & $\mathrm{mm}$ & 5 & 50 & 2 \\
D2 & Soil water deficit to start a new irrigation cycle & $\mathrm{mm}$ & 20 & 150 & 3 \\
I2 & Irrigation depth applied & $\mathrm{mm}$ & 5 & 50 & 2 \\
T3 & Accumulated thermal units to stop the irrigation & ${ }^{\circ}$ C.day & 1250 & 2000 & 5 \\
D3 & Soil water deficit to stop irrigation & $\mathrm{mm}$ & 20 & 150 & 3 \\
I3 & Irrigation applied at the last irrigation round & $\mathrm{mm}$ & 5 & 50 & 2 \\
\hline
\end{tabular}

Table 3 The eight parameters of the irrigation strategy to be optimized. min and max show the range of each parameter within which the optimum is sought. A step is the minimum feasible range of the parameter.

Some procedure parameters were set for all experiments. The maximum number of simulations was set to 2 million. $\beta$ - and $\operatorname{conc} \beta$-selections include a $20 \%$ probability of randomly choosing the promising region and the sampling follows a uniform distribution. As some of the partitioning alternatives need indices, we called them $\gamma r$ and $\gamma_{s}$ for the pivot partitioning, and $\gamma r$ for the maximum difference partitioning. We tested all possible combinations with $\gamma r \in\{0.2,0.5,0.8\}$ and $\gamma_{s} \in\{0.2,0.5,0.8\}$.

To compare alternatives, we defined some criteria in order to measure algorithm efficiency. Each time the direct margin average value increased, we stored it as well as the number of simulation runs used to achieve it. These stored values presented a strictly increasing curve in the simulation runs/direct margin criteria space. The general shape of this curve was made up of two distinct phases: one with a large improvement in direct margin average with a few simulation runs, and another with a tiny improvement with an infinite number of simulation runs. Therefore, we used simulation count and direct margin average corresponding to $95,97.5,99$ and $99.5 \%$ of the best value eventually achieved within 2 million of simulation runs. These indices allowed information about the best performances reached by the alternative to be kept, while focusing on the first increasing phase of interest. 
The number of runs criterion ensured that the algorithm efficiency does not depend on the computer's capabilities.

To recognize different techniques and their parameters, we used a $X Y Z$ code notation described in table 4 . The first item stands for selection alternative: $g s$ for greedy-selection, $b s$ for $\beta$-selection or $c s$ for conc $\beta$-selection. The second stands for partition alternative: $e p$ for equal-partition, $p p$ for pivot-partition or $m p$ for maximize_difference-partition. The last stands for the second evaluation index: $g e$ for global standard deviation $V_{g}, p 1 e$ for point standard deviation $V_{p 1}$ or $p 2 e$ for point standard deviation $V_{p 2}$ evaluation.

\begin{tabular}{|ll|ll|ll|}
\hline selection & $\mathrm{X}$ & division & $\mathrm{Y}$ & evaluation & $\mathrm{Z}$ \\
\hline$\beta$ & $b s$ & equal partition & $e p$ & average and global standard deviation & $g e$ \\
Conc $\beta$ & $c s$ & pivot partition & $p p$ & average and point standard deviation 1 & $p 1 e$ \\
Greedy & $g s$ & maximize difference & $m p$ & average and point standard deviation 2 & $p 2 e$ \\
\hline
\end{tabular}

Table 4 Triple code notation: definition of $X, Y$ and $Z$.

Alternative bs/ep/ge has been used as a test reference. We first compared separately selection, division and evaluation alternatives from $\mathrm{bs} / \mathrm{ep} / \mathrm{ge}$ to assess the impacts of single techniques. Then, we tested a few hybrid alternatives that seemed to be the most interesting according to previous observations.

\section{Results}

\subsection{Observation of $P 2$ alternatives}

\subsubsection{Selection alternatives}

Experimented selection alternatives are $\beta$ - (bs/ep/ge), conc $\beta$ - (cs/ep/ge) and greedy- (gs/ep/ge) selections.

Figure 4 displays the alternative averaged indices of 95, 97.5, 99 and 99.5\%. Alternative gs/ep/ge was faster and reached a slightly lower direct margin than bs/ep/ge. Alternative cslep/ge both achieved a lower direct margin and needed many more simulations to reach the best region. The gs/ep/ge's deviation along the number of simulation is the smallest.

\subsubsection{Division alternatives}

The tested partitioning alternatives are equal- (bs/ep/ge), pivot- (bs/pp/ge) and max_diff(bs/mp/ge) partitioning. We saw earlier (section 2.2) that $b s / p p / g e$ needs the thresholds $\gamma r$ and $\gamma_{s}$, and $\mathrm{bs} / \mathrm{mp} / \mathrm{ge}$ needs the threshold $\gamma \mathrm{r}$. Alternatives $\mathrm{bs} / \mathrm{mp} / g e_{-} 02$ and $\mathrm{bs} / \mathrm{mp} / g e_{0} 08$ respectively stand for $\mathrm{bs} / \mathrm{mp} / \mathrm{ge}$ with $\gamma r=0.2$ and $\mathrm{bs} / \mathrm{mp} / \mathrm{ge}$ with $\gamma r=0.8$. Alternative $b s / p p / g e \_08 \_02$ stands for the alternative $b s / p p / g e$ with $\gamma r=0.8$ and $\gamma_{s}=0.2$. Figure 5 displays the $b$ s/ep/ge and the non dominated alternatives.

bs/mp/ge_02 was faster but reached a slightly lower direct margin than bs/ep/ge. Both bs/mp/ge_08 and bs/pp/ge_08_02 reached a direct margin larger than bs/ep/ge with less runs. As alternatives are all very close, it is difficult to rank them. Figure 5 highlights the slight improvement due to partitioning methods, though $b s / p p / g e \_08 \_02$ can be considered as the best. Indeed its maximum value was at least as good as the others, while the minimum and average values were larger. 


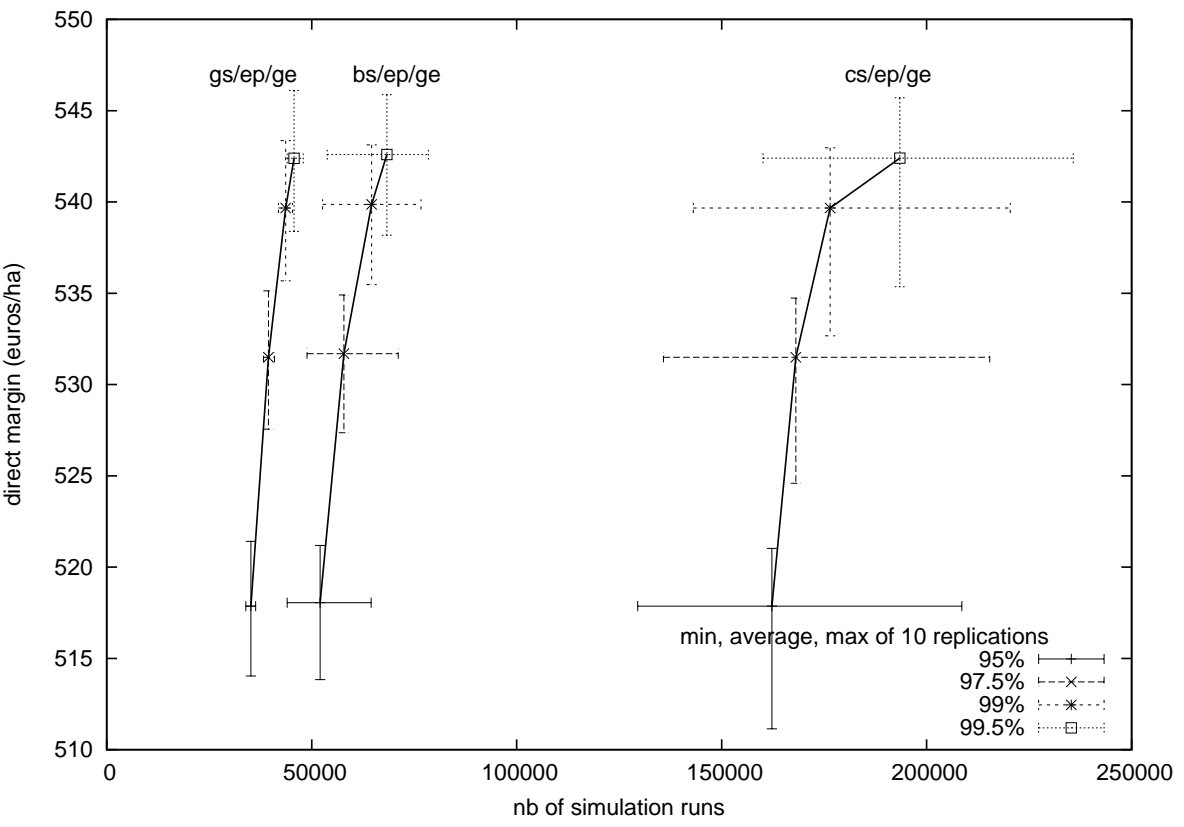

Fig. 4 Evolution of selection alternatives (10 replications each).

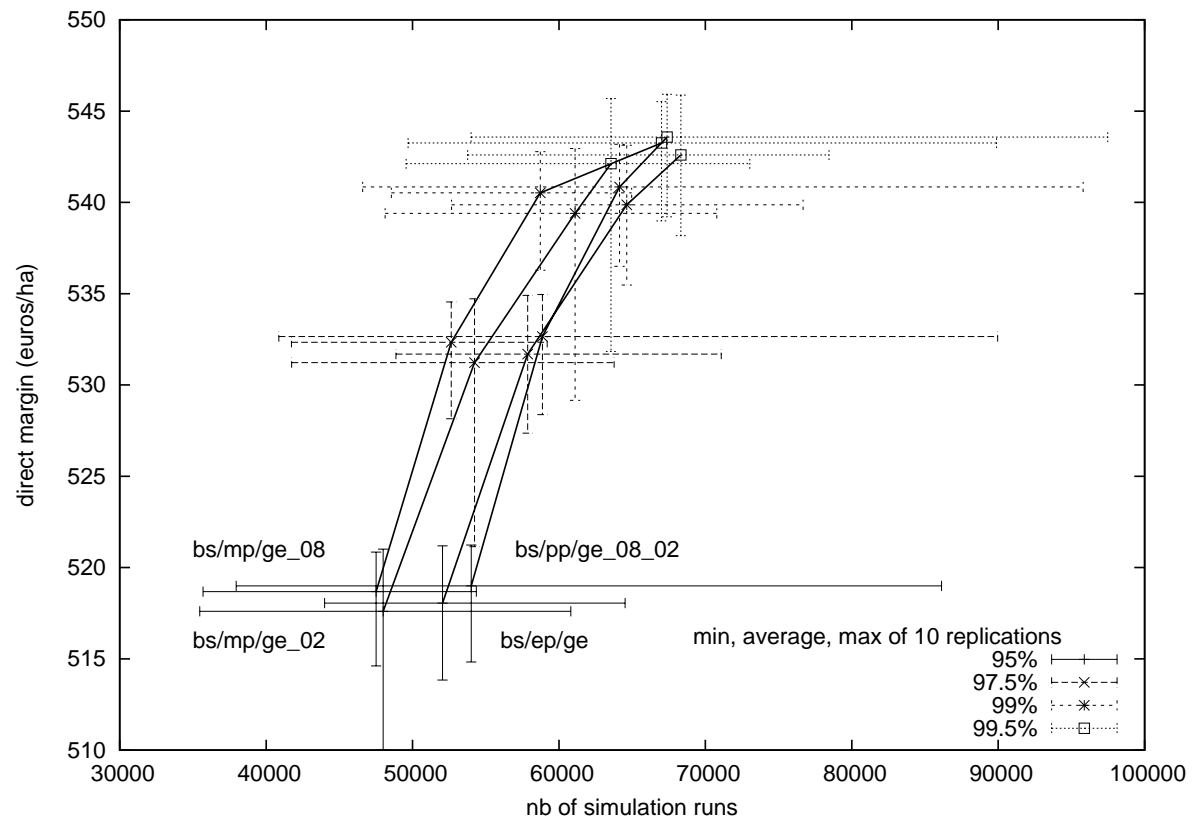

Fig. 5 Evolution of the best partitioning alternatives (10 replications each). 


\subsubsection{Evaluation alternatives}

The evaluation alternatives include the expected value of the sampled performance measures defined as score $_{1}$. score $_{2}$ stands either for the global standard deviation $V_{g}$ (bs/ep/ge), or for the sampling standard deviation $V_{p 1}(b s / e p / p 1 e)$, or for the sampling standard deviation $V_{p 2}$ (bs/ep/p2e) evaluations.

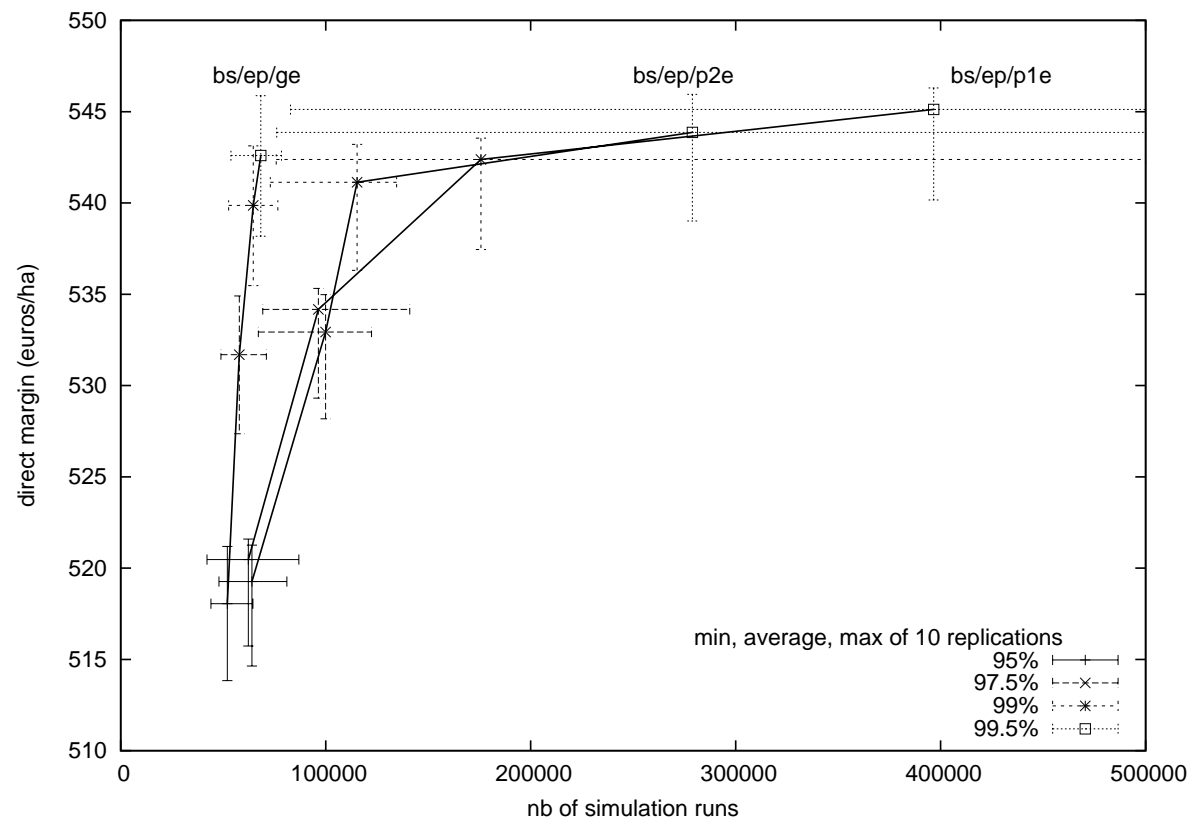

Fig. 6 Evolution of evaluation alternatives (10 replications each).

Figure 6 shows that $b s / e p / p 2 e$ and $b s / e p / p 1 e$ needed many more simulations than $b s / e p / g e$. Nevertheless, they both reached a larger averaged direct margin, which amount has never been reached with previous alternatives. The largest number of simulation runs (for $\mathrm{bs} / \mathrm{ep} / \mathrm{p} 1 \mathrm{e}$ ) was larger than 1.5 million of simulations. This large spread in simulation runs shows that bs/ep/ple reached its second phase of a small benefit for an infinite number of simulation runs. Although the direct margin performance was the best, this large spread means that it is difficult to forecast the required time to reach the optimal sub-region.

\subsubsection{Hybrid alternatives}

There are many hybrid alternatives. We decided to test a few of them, selecting those which could be efficient trade-off between simulation runs number and direct margin achieved. First we hybridized the fast $g s / e p / g e$ alternative with better partition techniques $\left(g s / p p / g e \_08 \_02\right.$ and $g s / m p / g e \_02$ ). Note that it is useless to couple $g s / e p / g e$ with other evaluation techniques since it does not take into account the score $_{2}$ value. Then we hybridized the alternatives with larger direct margin achievement with faster ones ( $b s / p p / p 1 e_{-} 08 \_02, b s / m p / p 1 e \_02$, $b s / p p / p 2 e_{2} 08 \_02$ and $\left.b s / m p / p 2 e \_02\right)$. Eight hybrid alternatives were eventually tested. 


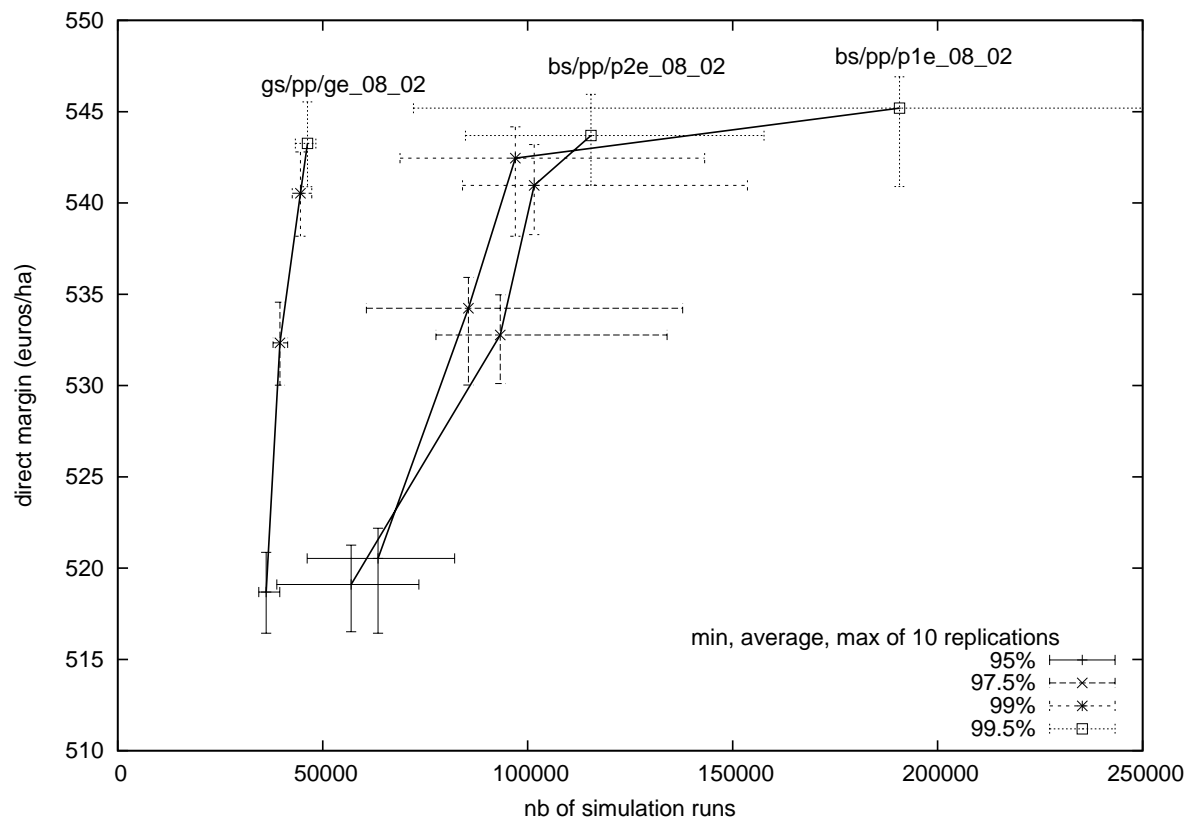

Fig. 7 Evolution of non dominated hybrid alternatives (10 replications each).

Figure 7 shows the hybrid alternatives that are non dominated with regard to simulation runs and $99.5 \%$ of the final direct margin achieved. Partitioning alternatives maximizing the difference of region indices have completely disappeared as they are always dominated by the division technique cutting the promising region upon the pivot.

\subsection{Observation of irrigation strategies}

In addition to algorithms results, we observed the input variable envelopes translating two computed irrigation alternatives. Figures 8 and 9 represent multiple axis charts of the envelopes enclosing the 10 optimal regions reached for the 10 replications run of the gs/ep/ge and $\mathrm{bs} / \mathrm{pp} / \mathrm{ple}$ alternatives. Couples of dashed lines bound the parameters of the optimal regions that were reached for every replications.

The shape envelopes of figures 8 and 9 are very similar. The main differences involve the $T 3$ parameter which still allows a wide range of possibilities. Input variables $D 3$ (soil water deficit to stop irrigation) and $I 3$ (amount of water applied during last irrigation round) have been barely broken down. On the other hand, the envelopes of input variables of $D 1$ (soil water deficit to start a new irrigation cycle) and $I 2$ (amount of water applied to a new irrigation round) are thin for every alternative.

The largest averaged direct margin reached over all replications is $547 € / h a$. It results from the alternative $b s / p p / p 1 e$ where the envelope denotes the following strategy. The first irrigation is started after observing a small water deficit, and a medium amount of water is applied. The new irrigations are performed as soon as a small water deficit is observed and 


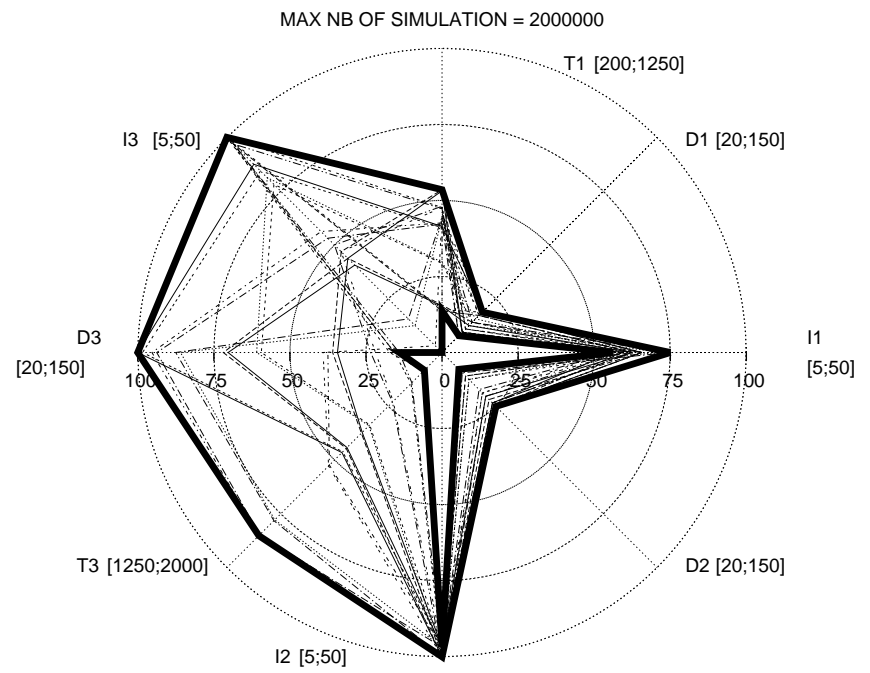

Fig. 8 Input variable envelope of the 10 optimal regions reached for the 10 replications of alternative gs/ep/ge.

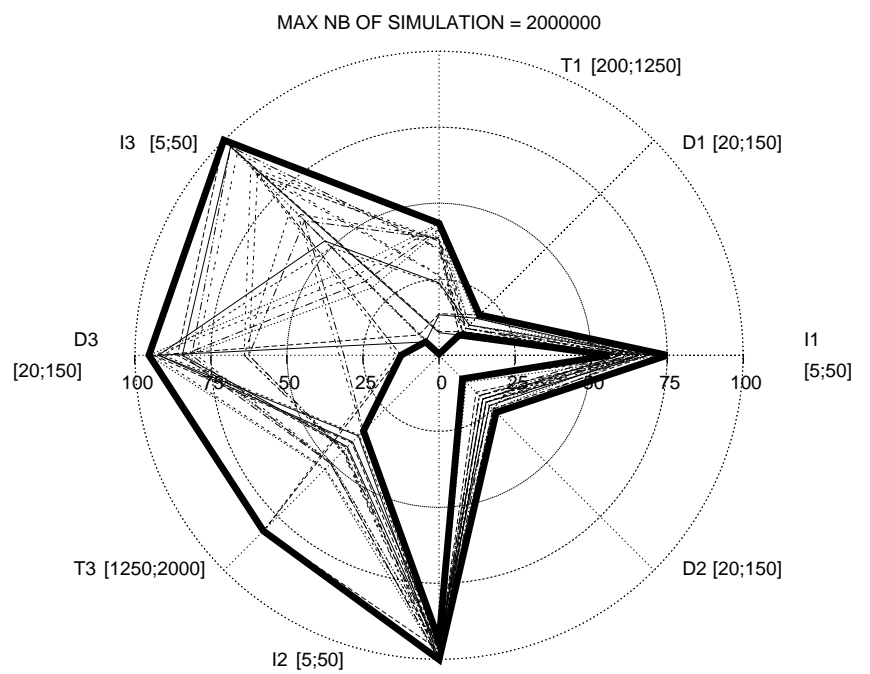

Fig. 9 Input variable envelope of the 10 optimal regions reached for the 10 replications of alternative $b s / p p / p 1 e$.

a large amount of water is applied. The temperature, water deficit and water amount leading to the last irrigation are very variable. 


\section{Discussion}

\subsection{P2 alternatives efficiency}

With regard to simulation runs and $99.5 \%$ of final direct margin achieved, the conc $\beta$ selection is completely dominated by the other alternatives. One can guess that the convex and evolutionary nature of the pending regions set makes the $\beta$-selection more efficient. Partitioning alternatives with dominated results have also been removed, so that only the alternatives including $\gamma r=0.8$ and $\gamma_{s}=0.2$ technique's parameters have been kept. This combination of indices expresses relatively high level of risk acceptance (low sampled decision vector index) and that the pivot was chosen to build large 'good' regions and small 'bad' ones (high level of region index). These selected alternatives are the most efficient at simulating the crop model MODERATO. Concerning the evaluation alternatives, the use of sampled point standard deviations achieved larger direct margins, but needed many more simulation runs. The cutting process does not noticeably improve the $P 2$ procedure, we nevertheless noticed that each of the non dominated hybrid alternatives included the pivot partitioning.

The alternatives shown in figure 10 compete to be the best irrigation strategy designer. The $g s / e p / g e$ and $g s / p p / g e$ alternatives were the most robust with respect to simulation runs. In addition, the $g s / p p / g e$ alternative had a direct margin robustness which was at least as good as those of the others.

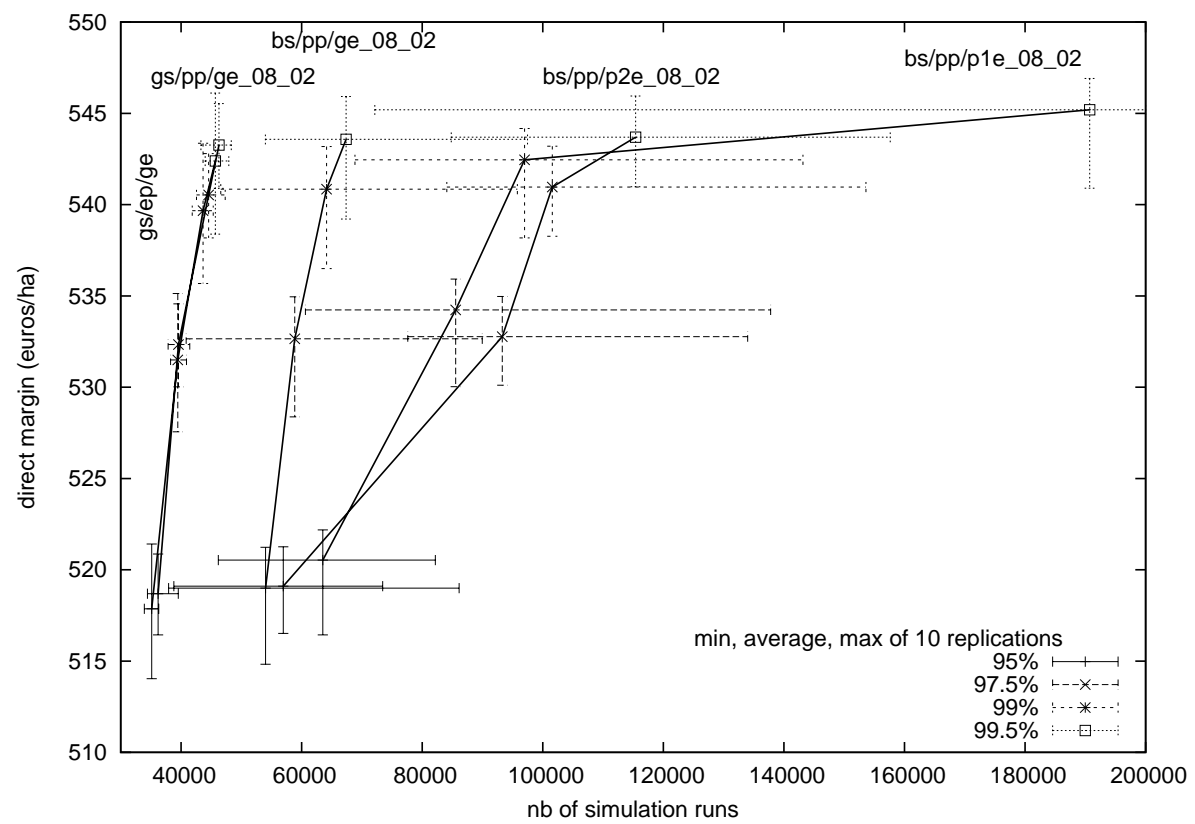

Fig. 10 All non dominated alternatives.

Figure 11 shows the frontier made of the $99.5 \%$ thresholds of the non dominated combinations. Combinations including the greedy selection are the fastest and need less than 


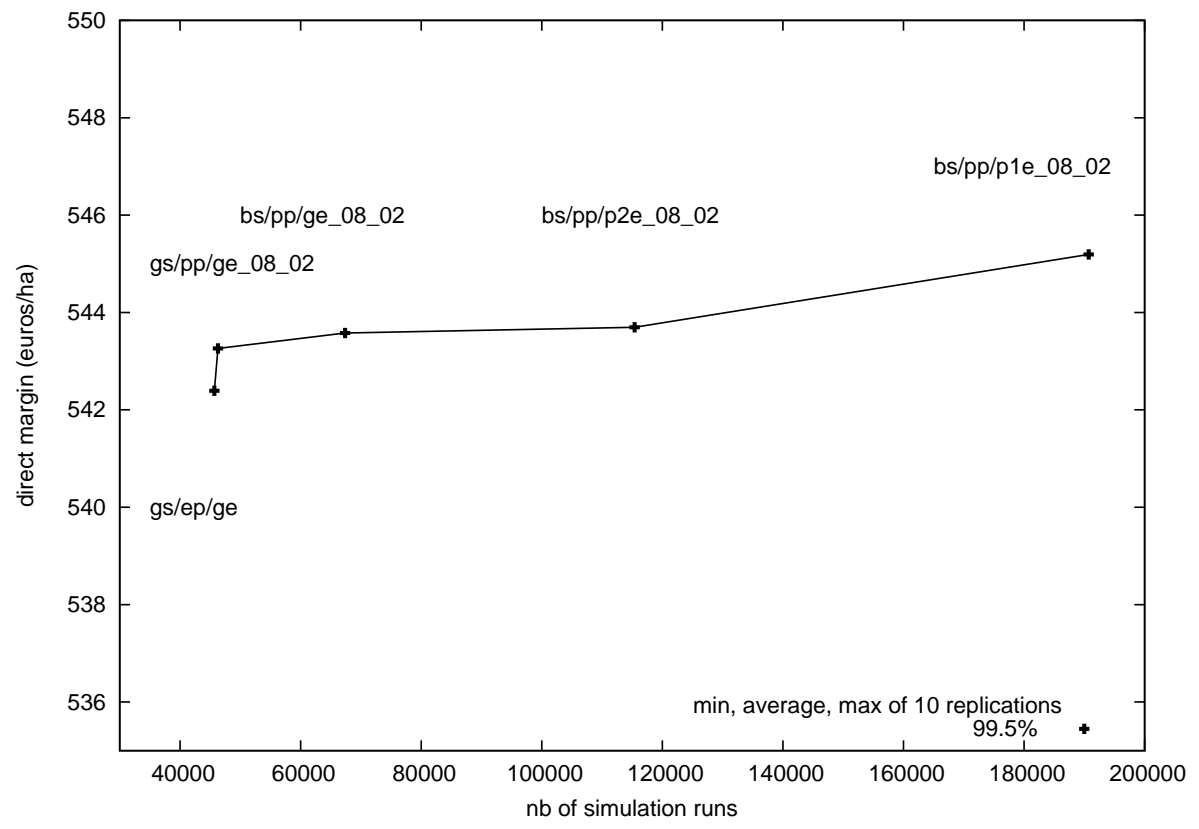

Fig. 11 All non dominated alternatives for $99.5 \%$ of the optimal region.

50000 of simulation runs to reach $99.5 \%$ of the optimal region. Combinations including $\beta$-selection achieve higher direct margins, but it requires a far larger amount of simulation runs. Considering the frontier shape, we regard the $g s / p p / g e$ alternative as an improvement over the $g s / e p / g e$ alternative. Although the three last alternatives improve the direct margin, too many simulation runs are needed to do so. The $\mathrm{gs} / \mathrm{pp} / \mathrm{ge}$ alternative is now the default combination of techniques included in the $P 2$ algorithm.

The best combination of techniques is not directly expendable to other stochastic optimization problems. However, the conclusions drawn about the different techniques would allow the user to set it easily, especially when making decision about cultural operations.

\subsection{Optimal irrigation plans}

This section deals with the irrigation plans produced with the $g s / p p / g e$ combination, compared with the irrigation plans produced with our reference combination $b s / e p / g e$.

Figure 12 shows the optimal decision space envelopes reached for two alternatives $\mathrm{gs} / \mathrm{pp} / \mathrm{ge}$ chosen as the best, and $\mathrm{bs} / \mathrm{ep} / \mathrm{ge}$ which was used as a test reference. The main noticeable difference still concerns the $T 3$ parameter, and although its range is still wide, its optimal value could be more difficult to find compared to the others. The recurrent thinness of input parameters $D 1$ and $D 2$ point out their sensitivity, while $T 3$ values variation are strongly related to the prior consumption of the limited amount of water. Nevertheless, the $g_{s} / \mathrm{pp} / \mathrm{ge}$ alternative improved both the direct margin reached and the number of simulation runs. The optimal decision space was at least no larger than our reference alternative. The agricultural case that was studied was the same as the one we used in Bergez et al (2004). 


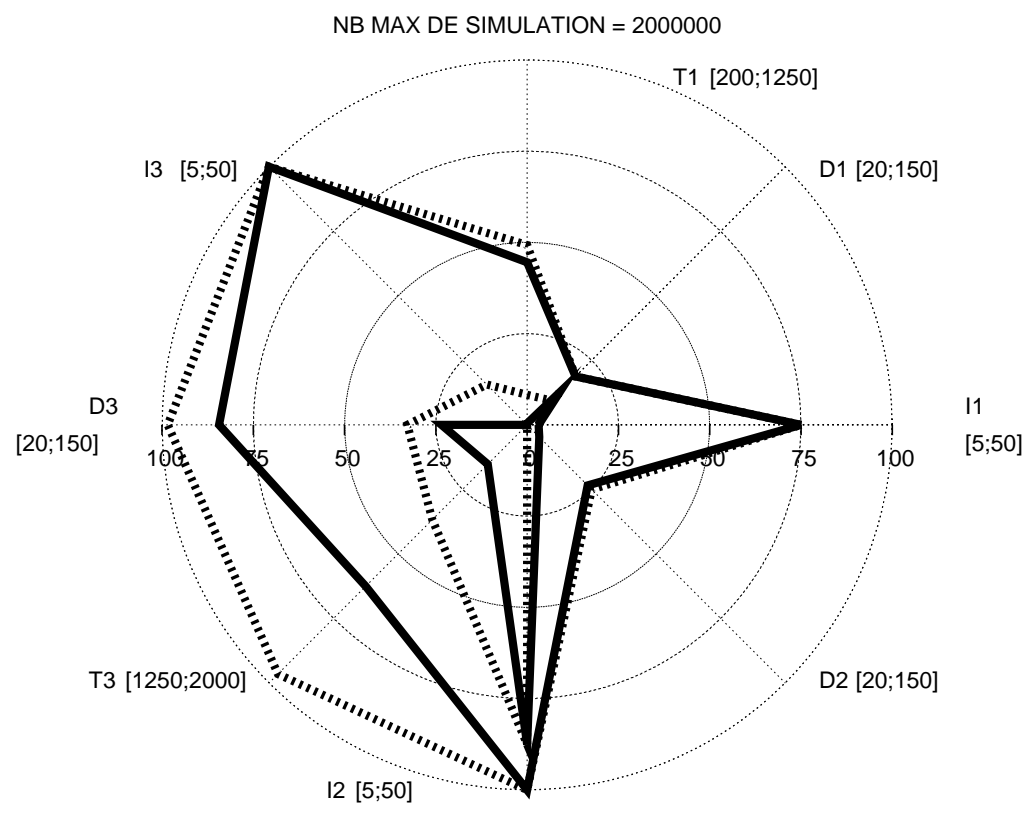

Fig. 12 Input variable envelope of the 10 optimal regions reached for the 10 replications of alternative gs/pp/ge_08_02 (solid line) and Input variable envelope of the 10 optimal regions reached for the 10 replications of alternative bs/ep/ge (dashed line).

The direct margin average of $534 € / h a$ was reached within 1600000 simulation runs with the $P 2 p$ algorithm. The direct margin average reached with the $g s / p p / g e \_08 \_02$ of the $P 2$ algorithm was $545 € / h a$ within 2000000 simulation runs, and the direct margin of $534 € / h a$ was achieved within less than 40500 simulation runs.

\subsection{Conclusion}

Although all the combinations are still available, we set the default $P 2$ algorithm as the combination of greedy selection, pivot partition and average evaluation. We reached this conclusion by testing different combinations with an 8-parameter irrigation design problem. These combinations included common and new techniques for the performance of the three main steps of the hierarchical decomposition procedure: selection, division and evaluation.

The aim of this paper was to investigate hierarchical decomposition of three main techniques in order to improve an earlier proposal rather than to compare it with other approaches. The optimal direct margin reached was about $10 € /$ ha higher than the one reached with the earlier proposal. About a quarter as many simulation runs were needed to reach the previously optimal direct margin. Hence the results obtained were satisfactory. We are now working on the extension of this approach for a better consideration of uncertainty, and of multiple objectives. In addition, more attention will be paid to the representation of the optimal decision space in order to facilitate its use by advisers. 


\section{References}

Andradóttir S (1998) A review of simulation optimization techniques. In: Proccedings of the 1998 Winter Simulation Conference, pp 151-158

Aubry C, Papy F, Capillon A (1998) Modelling decision-making processes for annual crop management. Agricultural Systems 56(1):45-65

Bergez JE, Debaeke P, Deumier J, Lacroix B, Leenhardt D, PLeroy, DWallach (2001a) Moderato: an object-oriented decision tool for designing maize irrigation schedules. Ecological Modelling 137:43-60

Bergez JE, Eigenraam M, Garcia F (2001b) Comparison between dynamic programming and reinforcement learning: A case study on maize irrigation management. Proceedings of the 3rd European Conference on Information Technology in Agriculture (EFITA01), Montpellier (FR) pp 343-348

Bergez JE, Garcia F, Lapasse L (2004) A hierarchical partitioning method for optimizing irrigation strategies. Agricultural Systems 80:235-253

Bergez JE, Garcia F, Wallach D (2006) Representing and optimizing management decisions with crop models, elsevier edn, Wallach, D.; Makowski, D.; Jones, J.W., pp 175-210

Breiman L (1996) Technical Note: Some Properties of Splitting Criteria. Machine Learning 24(1):41-47

Ehrgott M (2005) Multicriteria Optimization. Springer

FAO MG (2002) Water: precious and finite resource. Tech. rep., Food and Agriculture Organization of the United Nations

Fu M, Glover F, April J (2005) Simulation Optimization: A Review, New Developments, and Applications. In: Winter Simulation Conference, 2005 Proceedings of the, pp 83-95

Huyer W, Neumaier A (1999) Global optimization by multilevel coordinate search. Journal of Global Optimization 14(4):331-355

Jones D, Perttunen C, Stuckman B (1993) Lipschitzian optimization without the Lipschitz constant. Journal of Optimization Theory and Applications 79(1):157-181

Norkin V, Ermoliev Y, Ruszczynski A (1994) On Optimal Allocation of Indivisibles under Uncertainty. Operations Research 46(3):381-395

Ólafsson S, Kim J (2002) Simulation optimization. In: Winter Proccedings of the 2002 Winter Simulation Conference, pp 79-84

Papy F (2000) Farm models and decision support: a summary review. Research on agricultural systems: accomplishments, perspective and issues pp 89-107

Prasetio Y, Yen J, Zabinsky Z (2004) A probabilistic branch-and-bound scheme for simulation optimization. In: INFORMS annual meeting, Denver, USA

Shaffer M, Brodahl M (1998) Rule-based management for simulation in agricultural decision support systems. Computers and Electronics in Agriculture 21(2):135-152

Smith M (2000) The application of climatic data for planning and management of sustainable rainfed and irrigated crop production. Agricultural and Forest Meteorology 103(12):99-108

Wallach D, Goffinet B, Bergez J, Debaeke P, Leenhardt D, Aubertot J (2001) Parameter estimation for crop models a new approach and application to a corn model. Agronomy Journal 93(4):757-766 